“C 2019 IEEE. Personal use of this material is permitted. Permission from IEEE must be obtained for all other uses, in any current or future media, including reprinting/republishing this material for advertising or promotional purposes, creating new collective works, for resale or redistribution to servers or lists, or reuse of any copyrighted component of this work in other works." 


\title{
An Adaptive UAV Network for Increased User Coverage and Spectral Efficiency
}

\author{
Hasini Viranga Abeywickrama, Ying He, Eryk Dutkiewicz, Beeshanga Abewardana Jayawickrama \\ University of Technology Sydney, Global Big Data Technologies Centre \\ Email: \{Hasini.V.Abeywickrama\}@ student.uts.edu.au \\ \{Ying.He, Eryk.Dutkiewicz\}@uts.edu.au \\ \{beeshanga.jayawickrama\}@ericsson.com
}

\begin{abstract}
Unmanned Aerial Vehicles (UAVs) are fast becoming a popular choice in a variety of applications in wireless communication systems. UAV-mounted base stations (UAV-BSs) are an effective and cost-efficient solution for providing wireless connectivity where fixed infrastructure is not available or destroyed. We present a method of using UAV-BSs to provide coverage to mobile users in a fixed area. We propose an algorithm for predicting the user locations based on their mobility data and clustering the predicted locations, so that one UAV-BS would provide coverage to one user cluster. The proposed method, hence is similar to the UAV-BSs following the users to keep them under the coverage region. Simulation results show that the proposed method increases the user coverage by $47 \%-72 \%$ and increases the spectral efficiency by $43 \%-55 \%$ depending on the scenario and in addition, reduces the number of UAV-BSs required to provide coverage.
\end{abstract}

Index Terms-UAV, Mobile Users, Mobile Network Coverage, Spectral Efficiency

\section{INTRODUCTION}

Unmanned Aerial Vehicles (UAVs) have opened doors to many potential applications in wireless communication systems, due to their many desirable features, such as high manoeuvrability, cost-efficiency and ease of deployment. In particular, UAV-mounted mobile base stations (UAV-BSs) can be deployed to provide wireless connectivity in areas of urgent need, such as battlefields or disaster scenes [1]. One of the greatest advantages of this approach is that the UAV-BSs can be sent to a specific target location immediately without having to deploy any infrastructure [2]. Unlike terrestrial base stations (BSs), even those mounted on ground vehicles, UAV-BSs can be deployed in any location and move along any trajectory constrained only by their aeronautical characteristics [1].

A number of studies related to UAV-BSs have been carried out. The authors of [4] propose the optimal altitude for a UAV-BS to provide maximum radio coverage on ground. The authors of [1] propose an algorithm called 'Spiral method' for optimizing the placement of UAV-BSs to provide coverage to fixed ground users. Sharma et. al propose a solution for optimal deployment of multiple drones between the macro and small cell tiers for improving coverage and capacity of the entire system [5]. Yet in most of these scenarios the mobility of UAV-BSs is not considered, hovering UAV-BSs are deployed instead of flying UAV-BSs, the UAV-BS is set to hover without dynamically changing its position. In [2] the

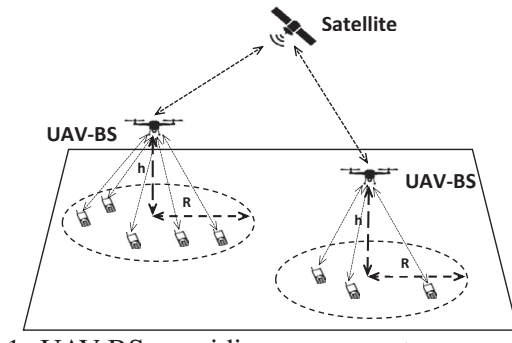

Fig. 1: UAV-BS providing coverage to ground users

authors consider dynamically repositioning the UAV-BSs to improve the performance. Yet, they do not take users' mobility into account. The authors of [3] consider user movement and aim to find the optimal placement for UAV-BS that aids the ground network. Hence, the scenario of mobile ground users being provided network coverage solely by UAV-BSs has not been widely researched. In this paper we consider the mobility of users and the manoeuvrability of UAV-BSs as well. We propose an algorithm where UAV-BSs follow the ground users based on their mobility patterns, so that the number of ground users covered by UAV-BSs is increased.

In this paper we consider a disaster struck area where coverage from terrestrial BSs is not available. UAV-BSs that are backhaul-connected via satellite links (We assume the backhaul has enough capacity to support all the active users), are used to provide wireless coverage to ground users who move within the considered area (Figure 1). We predict the future locations of the mobile users based on their current mobility data and cluster them based on their distances to each other, in a way that one UAV-BS would provide coverage to one user cluster. The user location prediction, user clustering and UAV-BS movement algorithms are presented later in this paper.

The rest of the paper is structured as below. Section II describes the system model and problem formulation, Section III presents the proposed adaptive network algorithm, Section IV gives the simulation results and Section V concludes the paper.

\section{System Model And Problem Formulation}

We consider a square area of width $l$, with $k$ mobile users who are denoted by $K=\{1,2, \ldots, k\}$. Their initial locations are distributed uniformly and at random. These initial locations 
are known and are given by $\left\{s_{k}\right\}_{k \in K}$, where $s_{k} \in \mathbb{R}^{2 \times 1}$ is the two dimensional (2D) coordinates of the $k$-th mobile user on the horizontal plane. The $k$ mobile users randomly move in the considered area according to the Random Waypoint (RWP) model [6].

UAV-BSs fly in the considered area aiming to provide coverage to the maximum number of mobile users. We assume that the UAVs fly in a fixed altitude of $h$. According to [7], $10 \mathrm{~m}$ is the optimal height for positioning a small cell BS. Lowering the antenna below $10 \mathrm{~m}$ would cause possible coverage issues and an antenna height higher than $10 \mathrm{~m}$ would increase interference with neighbouring cells [8]. Therefore, we consider a fixed height for the UAV-BSs and consider their movements restricted to a $2 \mathrm{D}$ plane.

We further assume that the transmit power of the UAV-BS is fixed $\left(P_{t x}\right)$ and the maximum allowed path loss $\left(P L_{\max }\right)$ at the receiver for reliable communication is given. For ground users, the path loss threshold can be considered a coverage disk of radius $R$, as all receivers inside this coverage disk would have a path loss less than or equal to $P L_{\max }$ [4].

We consider a probabilistic line of sight (LOS) model and use the probability of having a line of sight between user and the UAV-BS, proposed in [4], which is stated below.

$$
P_{L O S}(h, r)=\frac{1}{1+a \exp (-b[\theta-a])}
$$

where $P_{L O S}$ is the probability of LOS connection, $h$ is the relative flying altitude of the UAV-BS, $r$ is the distance between the ground user and the UAV's location projected on ground, $a$ and $b$ are statistical parameters that depend on the environment. $\theta$ is $\arctan (h / r)$ in degrees. Based on the basic theories of probability, the probability of having a non line of sight (NLOS) connection is $P_{N L O S}(h, r)=1-P_{L O S}(h, r)$.

We assume that the Doppler effect due to the mobility of the UAV-BS is compensated for based on existing techniques (eg. frequency synchronization using a phase-locked loop) [9] as done in [10].

The path loss in $\mathrm{dB}$ is as below,

$$
\eta(d)=A+10 \gamma \log _{10}(d)
$$

where $\eta$ is the path loss and $A$ is the path loss at reference distance $(1 \mathrm{~m}), \gamma$ is the path loss exponent. $\eta, A$ and $\gamma$ would take different values for LOS and NLOS communication scenarios. $A$ and $\gamma$ have been obtained from field tests in [11]. $d=\sqrt{h^{2}+r^{2}}$, is the distance between the UAV-BS and the ground user.

The received power of the $k$-th user $\left(S_{k}(d)\right)$ can be calculated using (3) below.

$$
S_{k}(d)=P_{t x} 10^{\frac{-\eta(d)}{10}}
$$

The spectral efficiency of the $k$-th user $\left(\mu_{k}\right)$ can be calculated as below. $N_{0}$ is the user equipment noise power.

$$
\mu_{k}=\log _{2}\left(1+\frac{S_{k}(d)}{N_{0}}\right)
$$

The spectral efficiency of a user considering both LOS and NLOS communication scenarios can be derived as below [2].

$$
\begin{aligned}
\mu_{k} & =P_{L O S}(h, r) \log _{2}\left(1+\frac{S_{k}^{L O S}(d)}{N_{0}}\right) \\
& +P_{N L O S}(h, r) \log _{2}\left(1+\frac{S_{k}^{N L O S}(d)}{N_{0}}\right)
\end{aligned}
$$

where symbols with $L O S$ indicate the values for LOS communication scenario and NLOS indicate values for NLOS communication scenario.

We divide the entire simulation time into steps of $\Delta t$. We assume the initial locations of the users are known and they remain static in the known locations until the UAV-BSs initially position themselves to provide coverage to the users. We cluster the users such that one UAV-BS would provide coverage to one cluster of users. Once the initial coverage is provided, the mobile users start moving randomly following the RWP model. We assume, once a user is under the coverage zone of a UAV-BS, the UAV is able to collect velocity information of the user. Based on the collected velocity information, we predict the locations of the users at next $\Delta t$. Based on the user location predictions, we repeat the clustering and UAV placement process throughout the entire simulation time.

In order to minimize cost, we aim to minimize the number of UAVs deployed. For this, minimal number of user clusters should be formed, in a way that one cluster would be covered by one UAV-BS. This reduces to the fact that one user cluster should be covered by a circle with radius $R$. (It should be noted that there is the possibility of some users being covered by more than one UAV-BS. In such scenarios, inter-cell interference should be addressed by proper channel assignment, UAV-BS power management etc. [1]).

If $U=\{1,2, \ldots, u\}$ is the number of UAV-BS to be deployed (hence the number of user clusters to be formed), the problem can be formulated as suggested in [1].

$$
P(1):\left\{\begin{array}{l}
\text { minimize }|U| \\
\text { s.t } \quad d_{u k} \leq R
\end{array}\right.
$$

where $|U|$ is the cardinality of set $U . d_{u k}$ is the distance between the mobile user $k$ and the position of the closest UAV$\mathrm{BS} u$ projected on ground. $R$ is the coverage radius of a UAV$\mathrm{BS}$. The above constraint makes sure that all the users are under the coverage of at least one UAV-BS.

$P(1)$, also known as the Geometric Disk Cover (GDC) problem [12] is NP hard in general [1]. The p-centre problem, which is closely related to GDC has the objective of finding $\mathrm{p}$ centres (UAV-BS locations) to cover all $K$ nodes. According to [1], p-centre problem is in general difficult to solve and requires high computational complexity.

In this paper, we propose a heuristic, computationally lowcost solution to $P(1)$, by clustering the users based on the distance to their neighbours. The clustering algorithm will be described in detail later in the paper. 


\section{Proposed Adaptive Network Algorithm}

We propose movement strategies for UAV-BSs to provide coverage to a maximum number of users despite the users' mobility. We propose

- Predicting user movement and locations

- Clustering users based on the proximity of their predicted locations

- Moving UAV-BSs to provide coverage to the clusters, in a way flying energy is low

\section{A. User Movement Prediction}

We assume the initial locations of the users are known and the users remain static in the initial locations until the UAVBSs initially position themselves to provide coverage to all users. Once the UAV-BSs are positioned the users randomly move within the considered area based on the RWP method.

RWP is the most commonly used mobility model in the ad hoc networking research community [13]. In this model a mobile node moves in a convex domain along a zigzag path, at each turning point the node chooses a new destination randomly and then moves toward it at a constant speed [6]. In the context of this paper, the mobile users pause for a random time at each turning point before choosing a new destination.

We assume that the UAVs are able to acquire information about the velocities of the users who are in their coverage zone. We divide the entire simulation time into steps of $\Delta t$. If the selected $\Delta t$ is small enough, based on RWP method, we can assume that if a user is moving in a specific velocity, during the next $\Delta t$, the user would still be moving in the same velocity or would be static in the current position.

If the user is static, based on RWP, in the next $\Delta t$ the user would still be static in the same position or would start moving in a random direction. Due to the randomness of the user's movement, predicting an area instead of a point where the user would possibly be, would increase the accuracy. Based on the preferred accuracy, the range can be calculated as below.

$$
Y=\left\{\begin{array}{c}
\left(x-x^{\prime}\right)^{2}+\left(y-y^{\prime}\right)^{2}=\left(V_{\max } \Delta t\right)^{2}, z=100 \\
\left(x-x^{\prime}\right)^{2}+\left(y-y^{\prime}\right)^{2}=\left(\frac{V_{\max }-V_{\min }}{100} z t\right)^{2} \\
0<z<100
\end{array}\right.
$$

where $Y$ is the area of possible positions of the user. $\left(x^{\prime}, y^{\prime}\right)$ is the current position of the static user and if $V$ is the possible speed of the user $V \in\left[V_{\min }, V_{\max }\right], z$ is the percentage of preferred accuracy.

\section{B. User Clustering}

Our objective is to cluster the users, first based on the initial locations then based on the predicted positions in a way that one UAV-BS would be able to provide coverage to one cluster. This reduces to one user cluster being able to be covered by a disk of radius $R$. Most of the standard clustering algorithms require the knowledge of the number of clusters before the formation. In the coverage scenario considered, it would be advantageous to keep the number of UAV-BS open and not limited, as one key objective would be providing coverage to the most number of mobile users.

After the users start moving based on RWP model, the user clustering is based on predicted locations which can be calculated as described in the previous subsection. However, for simplicity we assume that if a user is moving, during the next $\Delta t$ the user would be moving in the same velocity and if the user is static, the user would be static during the next $\Delta t$. Simulation results presented later in this paper show that this assumption does not affect the outcome considerably.

Algorithm 1: Algorithm for user clustering

Input: Mobile user set $K$ with known/predicted locations $\left\{s_{k}\right\}_{k \in K}, \mathrm{R}$

Output: Cluster centres $C$

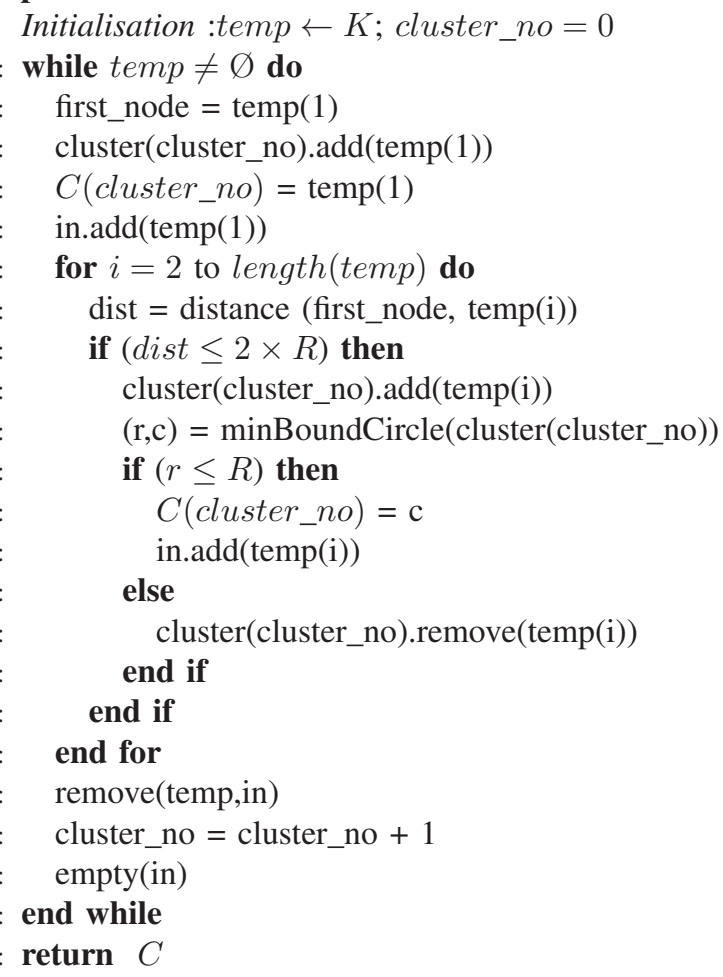

We cluster the ground users based on Algorithm 1. The function distance() would return the distance between the two points passed as arguments to the function, minBoundCircle() would return the radius $(r)$ and the centre $(c)$ of the smallest circle encircling the points passed to the function, remove() would remove the second subset from the first, passed as arguments. The function empty() would empty the array passed as an argument to it.

We cluster the users and their predicted locations based on Algorithm 1 , every $\Delta t$ for the entire period of simulation time.

\section{UAV-BS Movement}

Due to the distribution of the users based on their mobility, the number of clusters formed differs. The number of UAVBSs required to provide coverage changes accordingly within the span of simulation time. Because of the dynamic nature of the problem, we consider two scenarios 
- The available number of UAV-BSs is not restricted. We assume there are sufficient UAV-BSs to provide coverage to all the users

- The available number of UAV-BSs is limited. In this scenario, in order to provide coverage to the maximum number of users, we prioritize the clusters that have higher number of users

Algorithm 2: Algorithm for updating UAV-BS locations

Input: UAV-BS set with current locations $\left\{s_{u}(t=t)\right\}_{u \in U}$ cluster centres $C(t=t+\Delta t)$, available_UAVs

Output: $\left\{s_{u}(t=t+\Delta t)\right\}_{u \in U}$

Initialisation :to_cover $\leftarrow \mathrm{C}$; clear_UAV $\leftarrow s_{u}(t=t)$; cluster_no = length $(\mathrm{C})$

1: if (cluster_no $\leq$ available_UAVs) then

2: $\quad s_{u}(t=t+\Delta t)=$ position_UAVs(clear_UAV,to_cover)

3: else

4: $\quad$ diff $=$ cluster_no - available_UAVs

5: $\quad$ to_cover $=$ removeSmallest(to_cover,diff)

6: position_UAVs(clear_UAV,to_cover)

7: end if

8: return $\left\{s_{u}(t=t+\Delta t)\right\}$

Algorithm 2 is used for placing UAV-BSs. With Algorithm 2 we pick the UAV-BS closest to the next predicted cluster centre and move it to the centre of the specific cluster. This way we aim to reduce the energy spent on flying the UAVs, as this reduces the flying distance of each UAV-BS. The position_UAVs procedure is shown in Algorithm 3. The function removeSmallest makes sure that the clusters with the smallest number of users are removed from consideration and the number of available UAV-BSs and the number of considered user clusters match. The remove function behaves the same way as explained before. The procedure position_UAVs() is shown in Algorithm 3.

Algorithm 3: position_UAVs procedure

Input: set of cluster centres to be covered to_cover, set of available UAV-BSs clear_UAV

Output: $\left\{s_{u}(t=t+\Delta t)\right\}$

Initialisation :temp_cover $\leftarrow$ to_cover;

free_UAV $\leftarrow$ clear $U A V$

1: while temp_cover $\neq \varnothing$ do

$\mathrm{j}=$ getClosestUAV(temp_cover(1),free_UAV)

$\left\{s_{j}\right\}_{j \in U}(t=t+\Delta t)=$ temp_cover(1)

remove (free_UAV, j)

remove (temp_cover, temp_cover(1))

6: end while

7: return $\left\{s_{u}(t=t+\Delta t)\right\}$

\section{Simulation Results}

We tested the effectiveness of the proposed method based on the below criteria,

- Increase in user coverage

- Increase in spectral efficiency
- Reduction in number of deployed idle UAV-BSs (UAVBSs that are deployed but do not serve any users)

- Reduction in number of UAV-BSs required

Due to the lack of similar studies in literature, where the mobility of the UAV-BSs and the users is considered, we compare the proposed method (in terms of user coverage, number of deployed idle UAV-BSs and spectral efficiency) with the baseline scenario of having a fixed number of UAVBSs hovering in fixed locations covering the entire region considered. In the baseline scenario, the considered area is divided into equal cells and a UAV-BS is positioned at the centre of each cell.

Thus number of cells $(n)$, hence the number of UAV-BSs required in the baseline scenario is given by

$$
n=\left\lfloor\frac{l}{2 \times R}\right\rfloor^{2}
$$

where $l$ is the length of the square area to be covered, $R$ is the coverage radius of the UAV-BS.

We evaluated the algorithm based on the two scenarios of UAV-BS availability - when the number of available UAVBSs is not limited and when the number of available UAVBSs is limited. In the limited UAV-BSs number scenario, we assumed the number of available UAV-BSs to be the same as the number of UAV-BSs required in the baseline scenario of hovering UAV-BSs in fixed locations (given by (8)).

User coverage improvement was calculated as follows, where $U C I$ is the user coverage improvement, $\bar{X}_{i m p}$ is the average number of users covered using the proposed algorithm, $\bar{X}_{f i x}$ is the average number of users covered in the baseline scenario.

$$
U C I=\frac{\left(\bar{X}_{i m p}-\bar{X}_{f i x}\right) \times 100}{\bar{X}_{f i x}}
$$

In the proposed method, due to the errors in user location predictions, there is the possibility of deploying UAV-BSs that might not serve any users in reality. In the baseline scenario, due to the random movements and distribution of users, there is the possibility of having deployed UAV-BSs that do not provide coverage to any ground user. We compare the percentage of the deployed idle UAV-BSs in the two scenarios as a measurement of effectiveness.

In addition, we test the improvement of the spectral efficiency of the users based on the equation below.

$$
S E I=\frac{\left(\mu_{i m p}-\mu_{f i x}\right) \times 100}{\mu_{f i x}}
$$

where $S E I$ is the improvement in spectral efficiency, $\mu_{i m p}$ and $\mu_{f i x}$ represent the average spectral efficiency with the proposed algorithm and the baseline scenario.

One objective of the proposed method is to minimize the number of UAV-BSs required to provide coverage to ground users. To access this objective, we compared the proposed method with the 'Spiral method' proposed in [1]. Since the Spiral method is proposed for static users, the comparison is 
TABLE I: Simulation Parameters

\begin{tabular}{|l|l|l|}
\hline Symbol & Description & Value \\
\hline 1 & Area Width & $3.5 \mathrm{~km}$ \\
\hline$k$ & Number of Mobile Users & 30,50 \\
\hline$f$ & Working Frequency & $2 \mathrm{GHz}$ \\
\hline$P_{t x}$ & UAV-BS Transmitting Power & $24 \mathrm{dBm}$ \\
\hline$A$ & $\begin{array}{l}\text { Reference Distance Path Loss } \\
\text { (LOS/NLOS) }\end{array}$ & $41.1 / 33$ \\
\hline$\gamma$ & $\begin{array}{l}\text { Path Loss Exponent } \\
\text { (LOS/NLOS) }\end{array}$ & $2.09 / 3.75$ \\
\hline$N_{0}$ & UE Noise Power & $\begin{array}{l}-104 \\
{[14]}\end{array}$ \\
\hline$a, b$ & $\begin{array}{l}\text { Environmental Parameters for } \\
\text { Urban Area }\end{array}$ & $11.95,0.136$ \\
\hline$T$ & Simulation Time & $100 \mathrm{~s}$ \\
\hline$\Delta t$ & Time Slot & $0.1 \mathrm{~s}$ \\
\hline$h$ & UAV-BS Hovering Altitude & $10 \mathrm{~m}$ \\
\hline$R$ & Coverage Zone Radius & $0.5 \mathrm{~km}$ \\
\hline
\end{tabular}

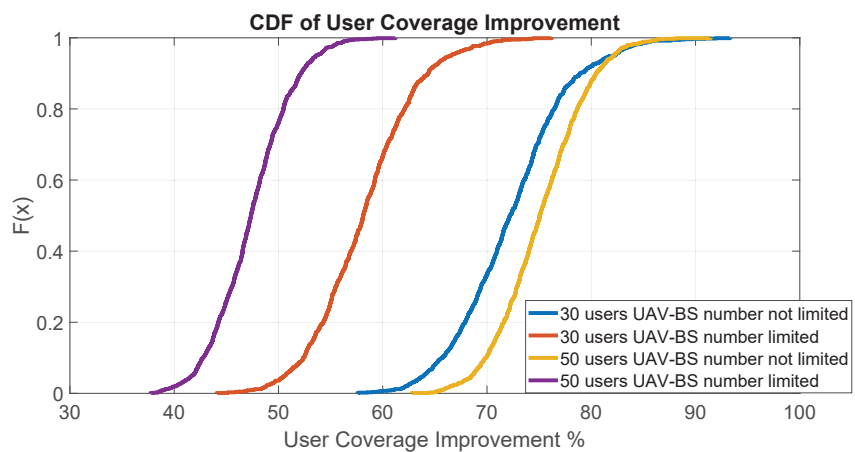

Fig. 2: User Coverage Gain

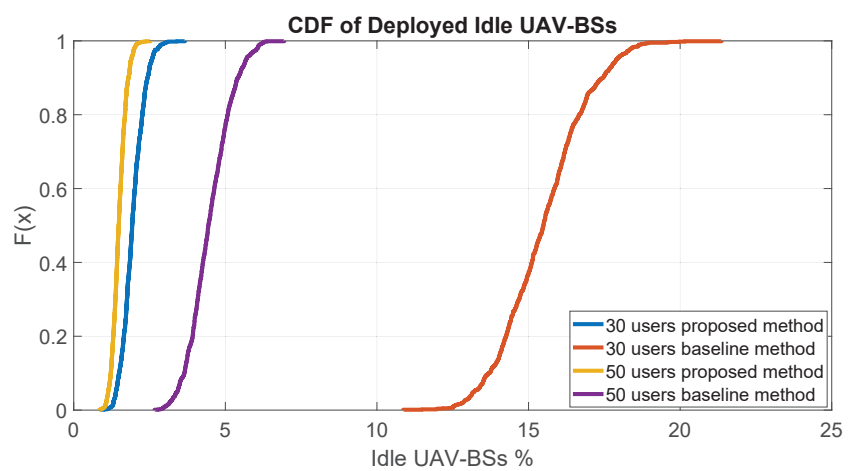

Fig. 3: Deployed Idle UAVs

made for the number of UAV-BSs required in the proposed method (with the number of UAV-BSs not limited), before the ground users start their movement based on RWP.

All results are based on simulations run in MATLAB. Each scenario has been run for 1000 iterations of 100 s simulation time. Unless otherwise mentioned the parameter values used for simulations are as shown in Table I.

The results shown by the graphs in Figure 2, Figure 3 and Figure 4 are summarized in Table II. As seen in Figure 2, the

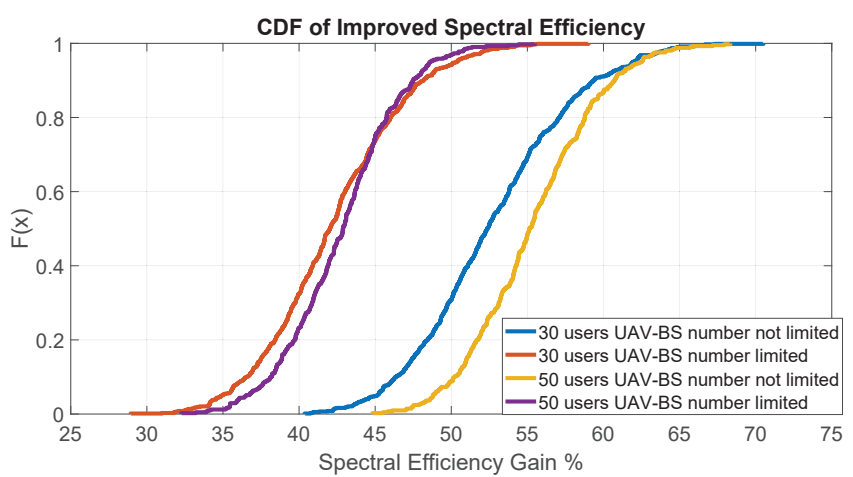

Fig. 4: Spectral Efficiency Gain

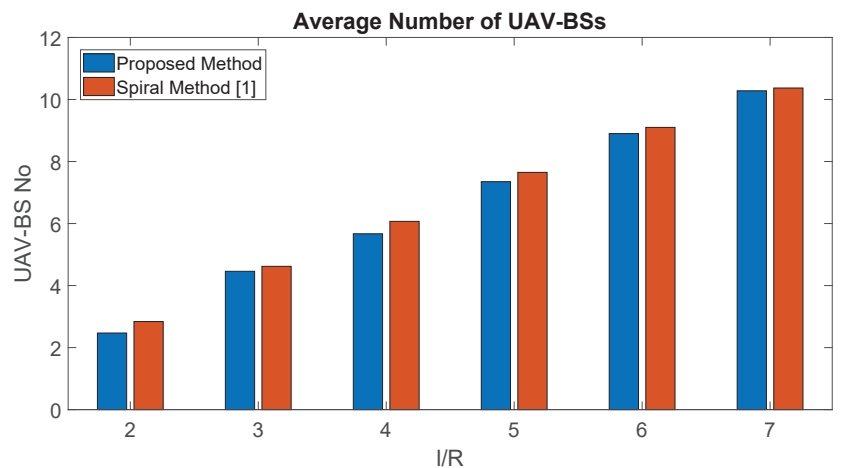

Fig. 5: Average Number of Required UAV-BSs

proposed method increases the user coverage by a considerable margin in both flexible number of UAV-BSs and limited number of UAV-BSs scenarios. With the proposed method, we aim to increase the number of covered users by adapting the network based on the user movements, as opposed to keeping the UAV-BSs stationed at fixed locations. This increases the percentage of covered users in each time interval.

Unlike stationing the UAV-BSs positioned at fixed locations, in the proposed method we propose deploying UAV-BSs based on the user demand. Thus, unlike the traditional scenario of positioning the UAV-BSs at fixed locations, in the proposed method UAV-BSs not having any users under their coverage zone is relatively low. This reduces the deployment of idle UAV-BSs (UAV-BSs that do not serve any users) as seen in Figure 3. The comparison made is Figure 3 is for the scenario when the number of UAV-BSs is not limited.

Since with the proposed method, the UAV-BSs follow the movements of the users, the distance between the users and the UAV-BSs reduces resulting in a considerable spectral efficiency. As Figure 4 shows, the spectral efficiency gain can increase up to an average of 55\% depending on the number of users and the availability of the UAV-BSs.

Figure 5 shows the number of UAV-BSs required to provide coverage to 30 ground users using the proposed method and 'Spiral method' [1]. The average number of required UAVBSs is shown against $l / R$ ratio. It can be seen that the number of required UAV-BSs is relatively low when employing the proposed method. The simulation results show an average 
TABLE II: Simulation Results

\begin{tabular}{|c|c|c|c|c|}
\hline \multicolumn{3}{|l|}{ Scenario } & \multirow{2}{*}{$\begin{array}{l}\text { Average Im- } \\
\text { provement or } \\
\text { Value } \\
72.3 \%\end{array}$} & \multirow{2}{*}{$\begin{array}{l}\text { Standard } \\
\text { Deviation }\end{array}$} \\
\hline \multirow[t]{4}{*}{ User Coverage } & \multirow[t]{2}{*}{$\begin{array}{l}30 \\
\text { users }\end{array}$} & $\begin{array}{l}\text { Flexible } \\
\text { number }\end{array}$ & & \\
\hline & & $\begin{array}{l}\text { Limited } \\
\text { number }\end{array}$ & $58.2 \%$ & 4.8 \\
\hline & \multirow[t]{2}{*}{$\begin{array}{l}50 \\
\text { users }\end{array}$} & $\begin{array}{l}\text { Flexible } \\
\text { number }\end{array}$ & $75.2 \%$ & 4.2 \\
\hline & & $\begin{array}{l}\text { Limited } \\
\text { number }\end{array}$ & $47.4 \%$ & 3.7 \\
\hline \multirow[t]{4}{*}{$\begin{array}{l}\text { Idle UAV-BS } \\
\text { (Value) }\end{array}$} & \multirow[t]{2}{*}{$\begin{array}{l}30 \\
\text { users }\end{array}$} & $\begin{array}{l}\text { Proposed } \\
\text { method }\end{array}$ & $1.9 \%$ & 0.4 \\
\hline & & $\begin{array}{l}\text { Baseline } \\
\text { method }\end{array}$ & $15.5 \%$ & 1.4 \\
\hline & \multirow[t]{2}{*}{$\begin{array}{l}50 \\
\text { users }\end{array}$} & $\begin{array}{l}\text { Proposed } \\
\text { method }\end{array}$ & $1.5 \%$ & 0.2 \\
\hline & & $\begin{array}{l}\text { Baseline } \\
\text { method }\end{array}$ & $4.5 \%$ & 0.7 \\
\hline \multirow[t]{4}{*}{$\begin{array}{l}\text { Spectral Effi- } \\
\text { ciency }\end{array}$} & \multirow[t]{2}{*}{$\begin{array}{l}30 \\
\text { users }\end{array}$} & $\begin{array}{l}\text { Flexible } \\
\text { number }\end{array}$ & $52.7 \%$ & 5.1 \\
\hline & & $\begin{array}{l}\text { Limited } \\
\text { number }\end{array}$ & $42.2 \%$ & 4.6 \\
\hline & \multirow[t]{2}{*}{$\begin{array}{l}50 \\
\text { users }\end{array}$} & $\begin{array}{l}\text { Flexible } \\
\text { number }\end{array}$ & $55.3 \%$ & 4.1 \\
\hline & & $\begin{array}{l}\text { Limited } \\
\text { number }\end{array}$ & $42.7 \%$ & 3.7 \\
\hline
\end{tabular}

decrease of $7 \%$ in comparison to the Spiral method.

\section{CONCLUSION}

UAV-BSs are a highly effective solution for providing wireless network coverage to users where coverage from a terrestrial BS is not available. In this paper, we propose a method of deploying UAV-BSs to provide coverage to mobile users. We aim to increase the number of users under coverage, by predicting and clustering the locations of the users. We exploit the manoeuvrability of the UAV-BSs and direct them along the user movements. The proposed method increases the user coverage by $47-72 \%$ depending on the number of ground users and UAV-BS availability while increasing the spectral efficiency by $43-55 \%$ depending on the scenario. The method proposed in this paper reduces the number of UAV-BSs required to provide coverage to ground users by an average of $7 \%$ in comparison to the baseline solution.

\section{REFERENCES}

[1] J. Lyu, Y. Zeng, R. hang and T. J. Lim, "Placement Optimization of UAVMounted Mobile Base Stations," IEEE Communications Letters, VOL. 21, NO. 3, Mar. 2017

[2] A. Fotouhi, M. Ding and M. Hassan, "Dynamic Base Station Repositioning to Improve Performance of Drone Small Cells," IEEE Globecom Workshops Dec. 2016.

[3] R. Ghanavi, E. Kalantariy, M. Sabbaghian, H. Yanikomerogluz, and Abbas Yongacoglu, "Efficient 3D Aerial Base Station Placement Considering Users Mobility by Reinforcement Learning," IEEE Wireless Communications and Networking Conference (WCNC) Apr. 2018

[4] A. Al-Hourani, S. Kandeepan and S. Lardner, "Optimal LAP Altitude for Maximum Coverage,” IEEE Wireless Communications Letters, Jul. 2014 pp $569-572$

[5] V. Sharma, M. Bennis, and R. Kumar, "UAV-assisted heterogeneous networks for capacity enhancement, IEEE Communications Letters, vol 20, no. 6, pp. 12071210, June 2016.

[6] E. Hyytia, Pa. Lassila and J. Virtamo, "Spatial Node Distribution of the Random Waypoint Mobility Model with Applications", IEEE Transactions on Mobile Computing, Vol.5, No.6, Jun. 2006
[7] M. Ding and D. L. Perez, "Please lower small cell antenna heights in $5 \mathrm{G}$," in 2016 IEEE Global Communications Conference (GLOBECOM), Dec. 2016, pp. 16.

[8] A. Fotouhi, M. Ding and M. Hassan, "DroneCells: Improving 5G Spectral Efficiency using Drone-mounted Flying Base Stations," Journal of Transactions on Mobile Computing, Jul. 2017.

[9] M. Umberto, "Synchronization Techniques for Digital Receivers", Springer Science \& Business Media, 2013

[10] U. Challita, W. Saad and C. Bettstetter, "Deep Reinforcement Learning for Interference-Aware Path Planning of Cellular-Connected UAVs," IEEE International Conference on Communications (ICC), May 2018

[11] 3GPP, "3GPP TR 36.828 version 11.0.0, release 11: 3rd generation partnership project; further enhancements to LTE time division duplex (TDD) for downlink-uplink (DL-UL) interference management and traffic adaptation," 3GPP Technical Report, Tech. Rep., 2012.

[12] A. Srinivas, G. Zussman, and E. Modiano, "Construction and maintenance of wireless mobile backbone networks," IEEE/ACM Trans. Netw., vol. 17, no. 1, pp. 239252, Feb. 2009.

[13] P. Lassila, E. Hyytia and H. Koskinen, "Connectivity Properties of Random Waypoint Mobility Model for Ad Hoc Networks”, Fourth Annual Mediterranean Workshop Ad Hoc Networks (Med-Hoc-Net 2005), Jun. 2005.

[14] X. Sun, N. Ansari "Jointly Optimizing Drone-Mounted Base Station Placement and User Association in Heterogeneous Networks," IEEE International Conference on Communications (ICC), May 2018 